\title{
NEGATIVE THEOREMS ON MONOTONE APPROXIMATION
}

\author{
JOHN A. ROULIER
}

\begin{abstract}
In this paper we show that for $f$ continuous on $[-1,+1]$ and satisfying $\left(f\left(x_{2}\right)-f\left(x_{1}\right)\right) /\left(x_{2}-x_{1}\right) \geqq \delta>0$, it is possible to have infinitely many of the polynomials of best uniform approximation to $f$ not increasing on $[-1,+1]$.
\end{abstract}

1. Introduction. Monotone approximation in its simplest form is the study of the uniform approximation of continuous functions on a closed interval by algebraic polynomials which are increasing there. Of particular interest is the case when $f$ is continuous and increasing on the interval since in this case $f$ may be uniformly approximated as close as desired by polynomials which are also increasing on the interval.

We now introduce some notation. We will work on the closed interval $[-1,+1]$ since no loss of generality is imposed by this. If $f \in C[-1,+1]$ we define the uniform norm by

$$
\|f\|=\max \{|f(x)| ;-1 \leqq x \leqq 1\} .
$$

For each nonnegative integer $n$ define

$$
\begin{aligned}
& H_{n}=\{p ; p \text { is an algebraic polynomial of degree less than or equal to } n\}, \\
& M_{n}=\left\{p \in H_{n} ; p^{\prime}(x) \geqq 0 \text { for all } x \text { in }[-1,+1]\right\} .
\end{aligned}
$$

Now define the degree of approximation

$$
E_{n}(f)=\inf \left\{\|f-p\| ; p \in H_{n}\right\}
$$

and the degree of monotone approximation

$$
D_{n}(f)=\inf \left\{\|f-p\| ; p \in M_{n}\right\} .
$$

It is well known that for each $f$ in $C[-1,+1]$ and for each nonnegative integer $n$ that there is a unique $p \in H_{n}$ such that $\|f-p\|=E_{n}(f)$. It is also known that there is a unique $q \in M_{n}$ such that $\|f-q\|=D_{n}(f)$. See G. G. Lorentz and K. L. Zeller [4]. The polynomials $p$ and $q$ above are called the polynomial of best approximation and the monotone polynomial of best approximation to $f$ respectively.

One might ask if the study of these concepts is trivial if $f$ is increasing. That is, if $f$ is increasing then is $p=q$ ? Or equivalently is $E_{n}(f)=D_{n}(f)$ ? The answer to these questions is in general no. Numerous examples are given to

Received by the editors January 13, 1975.

AMS (MOS) subject classifications (1970). Primary 41A10, 41A25.

1976, American Mathematical Society 
verify this. See J. A. Roulier [6] and [7] and G. G. Lorentz [3] and G. G. Lorentz and K. L. Zeller [5]. These references show that even if $n$ is large we need not have $D_{n}(f)=E_{n}(f)$. All of these examples have one thing in common:

There is no $\delta>0$ for which

$$
\begin{aligned}
\frac{f\left(x_{2}\right)-f\left(x_{1}\right)}{x_{2}-x_{1}} \geqq \delta \text { for all } x_{2} \neq x_{1} \text { in }[-1,+1] \\
\quad\left(\text { or } f^{\prime}(x) \geqq \delta \text { if } f \in C^{1}[-1,+1]\right) .
\end{aligned}
$$

On the other hand, J. A. Roulier in [7] proves:

THEOREM 1.1. If $f \in C^{2}[-1,+1]$, and if there is $\delta>0$ so that $f^{\prime}(x) \geqq \delta$ $>0$ for $-1 \leqq x \leqq 1$, and if $f^{\prime \prime} \in \operatorname{Lip} \alpha$ for some $0<\alpha \leqq 1$, then, for $n$ sufficiently large, $E_{n}(f)=D_{n}(f)$.

The purpose of this paper is to show that if $f$ satisfies (1), it still may happen that $D_{n}(f) \neq E_{n}(f)$ even for large $n$.

2. A negative theorem. The main theorem of this section involves the modulus of continuity of the $2 \pi$-periodic function $\phi(t)=f(\cos t)$ induced by $f\left(\omega(\phi, h)=\sup \left\{\left|\phi(t)-\phi\left(t^{\prime}\right)\right| ;\left|t-t^{\prime}\right| \leqq h\right\}\right)$.

THEOREM 2.1. Let $f$ be monotone increasing and continuous on $[-1,+1]$ and let $\phi(t)=f(\cos t)$. Assume that

$$
\limsup _{k \rightarrow \infty} \frac{k \omega(\phi, 1 / k)}{\log k}=+\infty .
$$

Then there are infinitely many positive integers $n$ for which the polynomial $p$ in $H_{n}$ of best approximation to $f$ is not increasing.

In order to prove this theorem we need two lemmas.

Given a continuous $2 \pi$-periodic function $g$ we define $E_{n}^{*}(g)$ to be the degree of approximation to $g$ by trigonometric polynomials of degree $n$ or less. The following lemma is due to S. B. Stečkin [8]. See also the book of G. G. Lorentz [2, p. 59].

LEMMA 2.2. There is a constant $M$ such that for each continuous $2 \pi$-periodic function $g$

$$
\omega(g, h) \leqq M h \sum_{0 \leqq n \leqq h^{-1}} E_{n}^{*}(g) .
$$

The next lemma makes use of (3).

LEMMA 2.3. Let $g$ be a continuous $2 \pi$-periodic function for which

$$
\limsup _{k \rightarrow \infty} \frac{k \omega(g, 1 / k)}{\log k}=+\infty
$$

Then there does not exist a positive constant $C$ so that $E_{n}^{*}(g) \leqq C / n$ for $n=1$, $2,3, \ldots$. 
Proof. Assume that there is $C$ so that

$$
E_{n}^{*}(g) \leqq C / n, \quad n=1,2,3, \ldots
$$

Then by Lemma 2.2 with $h=1 / N$ we have a constant $M$ for which

$$
N \omega\left(g, \frac{1}{N}\right) \leqq M \sum_{n=0}^{N} E_{n}^{*}(g) .
$$

But by the above assumption we have

$$
\sum_{n=0}^{N} E_{n}^{*}(g) \leqq E_{0}^{*}(g)+C \sum_{n=1}^{N} \frac{1}{n} \leqq D+2 C \log M \quad \text { for } N \geqq 3 .
$$

$D$ depends on $g$ but not on $N$.

Thus for $N$ sufficiently large

$$
\sum_{n=0}^{N} E_{n}^{*}(g) \leqq 3 C \log N .
$$

This together with (5) gives for $N$ sufficiently large

$$
N \omega(g, 1 / N) / \log N \leqq 3 C M .
$$

But this contradicts (4).

Hence, the assumption is false and the lemma is proven.

Proof of THEOREM 2.1. Assume that $f$ satisfies the hypotheses of the theorem and that there is $N>0$ so that for all $n \geqq N$ the $p \in H_{n}$ of best approximation to $f$ is increasing. Let $n \geqq N$ be given. It follows from the Chebyshev alternation theorem (Lorentz [2, p. 30]) that there are $n$ +2 points $x_{0}<x_{1}<\cdots<x_{n+1}$ in $[-1,+1]$ where $\left|f\left(x_{i}\right)-p\left(x_{i}\right)\right|=E_{n}(f)$ and $f\left(x_{i}\right)-p\left(x_{i}\right)$ alternate in sign. If $p\left(x_{i+1}\right)=f\left(x_{i+1}\right)-E_{n}(f)$ then $p\left(x_{i}\right)$ $=f\left(x_{i}\right)+E_{n}(f)$ and

$$
p\left(x_{i+1}\right)-p\left(x_{i}\right)=f\left(x_{i+1}\right)-f\left(x_{i}\right)-2 E_{n}(f) .
$$

By our assumption that $p$ is increasing, we must have

$$
E_{n}(f) \leqq\left(f\left(x_{i+1}\right)-f\left(x_{i}\right)\right) / 2
$$

for all such $i$. Since there are at least $[(n+1) / 2]$ such pairs of points we have

$$
\begin{aligned}
\frac{n}{2} E_{n}(f) & \leqq\left[\frac{n+1}{2}\right] E_{n}(f) \leqq \frac{1}{2} \sum\left(f\left(x_{i+1}\right)-f\left(x_{i}\right)\right) \\
& \leqq \frac{1}{2}(f(1)-f(-1)) .
\end{aligned}
$$

Thus for $n \geqq N$

$$
E_{n}(f) \leqq(f(1)-f(-1)) / n \text {. }
$$

Now $E_{n}^{*}(\phi)=E_{n}(f)$ where $\phi(t)=f(\cos t)$. Thus (6) gives

$$
E_{n}^{*}(\phi) \leqq(f(1)-f(-1)) / n \text { for } n \geqq N \text {. }
$$


But this contradicts Lemma 2.3 since (7) implies the existence of a constant $C$ for which $E_{n}^{*}(\phi) \leqq C / n$ for $n=1,2,3, \ldots$ Thus the assumption is false and the theorem is proven.

It is clear from the above proof that the following stronger but less constructive theorem is true.

THEOREM 2.4. Let $f$ be continuous and monotone increasing on $[-1,+1]$ and assume that $E_{n}(f) \neq O(1 / n)$. Then there are infinitely many positive integers $n$ for which the polynomial $p$ in $H_{n}$ of best approximation to $f$ is not increasing.

3. Further negative results. In the previous section we showed that all continuous increasing functions whose moduli of continuity satisfied a certain condition would fail to have increasing polynomials of best approximation even for $n$ large. One might ask if $f$ satisfies (1) but not (2) whether for $n$ large enough the polynomials of best approximation must be increasing.

The purpose of this section is to provide a constructive proof that this need not happen no matter how nice the modulus of continuity of $f$ is.

THEOREM 3.1. Let $\omega$ be any modulus of continuity. Then there is ancreasing function $f$ in $C[-1,+1]$ which satisfies (1) and for which

$$
\omega(h) \leqq \omega(f, h) \leqq K \omega(h),
$$

and yet there are infinitely many positive integers $n$ for which the polynomial of best approximation from $H_{n}$ to $f$ is not increasing.

In order to prove this theorem we need several lemmas.

The following two lemmas are found in Lorentz [2, p. 45 and p. 94 respectively].

LeMma 3.2. Let $\omega$ be any modulus of continuity. Then there is a concave modulus of continuity $\bar{\omega}$ with the same domain of definition as $\omega$ for which

$$
\frac{1}{2} \bar{\omega}(h) \leqq \omega(h) \leqq \bar{\omega}(h)
$$

LEMMA 3.3. If $g(x)=|x|$ on $[-1,+1]$ then there is a constant $M>0$ independent of $n$ for which

$$
E_{n}(g) \geqq M / n, \quad n=1,2, \ldots
$$

The next lemma is due to M. I. Kadec [1]. We first make a few comments.

Let $\phi$ be a $2 \pi$-periodic continuous even function, and for each positive integer $n$ let $T_{n}$ be the $n$th degree trigonometric polynomial of best approximation to $\phi$. Then $T_{n}$ is an even trigonometric polynomial.

By the Chebyshev alternation theorem then there are $2 n+2$ points in $[-\pi, \pi]$ at which $\phi-T_{n}$ assumes its maximum absolute value with alternating signs. This together with the fact that $\phi$ and $T_{n}$ are even shows the existence of at least $n+2$ points $t_{0, n}<t_{1, n}<\cdots<t_{n+1, n}$ in $[0, \pi]$ of maximum deviation at which the signs alternate. Let

$$
\Delta_{n}=\max _{0 \leqq k \leqq n+1}\left|t_{k, n}-\frac{k \pi}{n+1}\right| .
$$


LemMA 3.4. Let $\phi$ be a continuous even $2 \pi$-periodic function and let the notation be as above, and let $\varepsilon>0$ be given. Then

$$
\liminf _{n \rightarrow \infty} \Delta_{n} n^{\frac{1}{2}-\varepsilon}=0
$$

The following corollary to Lemma 3.4 will be used in the sequel.

Corollary. Let $f \in C[-1,+1]$ and for each $n=0,1,2, \ldots$ let $x_{0, n}<\ldots$ $<x_{n+1, n}$ be a Chebyshev alternation for $f$. Let

$$
\delta_{n}=\max _{0 \leqq k \leqq n+1}\left|x_{k, n}-\cos \left(\frac{k \pi}{n+1}\right)\right| .
$$

Then there is a sequence $\left\{n_{j}\right\}_{j=0}^{\infty}$ of positive integers for which

$$
\lim _{j \rightarrow \infty} \delta_{n_{j}}=0 .
$$

Proof. Let $\phi(t)=f(\cos t)$ and use Lemma 3.4.

Proof of Theorem 3.1. Let $M$ be as in (10) and let $\varepsilon=\min (1, M / 10)$. Let $\omega$ be any modulus of continuity. We define

$$
f(x)= \begin{cases}x-\omega(1)+\omega(x+2) & \text { on }[-2,-1], \\ 2 x+|x| & \text { on }[-1,+1], \\ 3+\varepsilon(x-1) & \text { on }[1,2] .\end{cases}
$$

Clearly $f \in C[-2,2]$ and is increasing and satisfies (1) on $[-2,2]$ with $\delta$ $=\min (1, \varepsilon)$. We will work on $[-2,+2]$ for simplicity of notation although everything could be "shrunk" to $[-1,+1]$ with no difficulty.

Clearly for $0 \leqq h \leqq 1$

$$
\omega(h) \leqq \omega(f, h) \leqq 4 h+\omega(h) .
$$

Let $\bar{\omega}$ be a concave modulus of continuity for which (9) holds. It is easy to see that $\bar{\omega}(h) / h$ is a decreasing function of $h$ (Lorentz [2, p. 44]). Thus for $0<h \leqq 1, \bar{\omega}(h) / h \geqq \bar{\omega}(1)$. Hence, for $0 \leqq h \leqq 1$,

$$
h \leqq C_{1} \bar{\omega}(h) \text {. }
$$

Thus using (9), (14) and (15) we have for $0 \leqq h \leqq 1$

$$
\omega(h) \leqq \omega(f, h) \leqq K \omega(h),
$$

where $K=8 C_{1}+1$.

Let $g(x)=2 x+|x|$ on $[-1,+1]$. Then for $n \geqq 1, E_{n}(g)=E_{n}(|x|)$. Thus (10) gives

$$
E_{n}(g) \geqq M / n \quad \text { for } n=1,2, \ldots
$$

Let $E_{n}(f)$ be the best approximation to $f$ by polynomials from $H_{n}$ on $[-2,+2]$. It is easy to see that for $n=0,1,2, \ldots, E_{n}(g) \leqq E_{n}(f)$. This combines with (17) to give 


$$
E_{n}(f) \geqq M / n \text { for } n=1,2,3, \ldots .
$$

Now use the corollary to Lemma 3.4 to find a sequence $\left\{n_{j}\right\}_{j=0}^{\infty}$ of positive integers so that (13) holds. We note that we are using this corollary on $f$ on the interval $[-2,2]$. This is accomplished by first applying the corollary to $f(2 z)$ for $-1 \leqq z \leqq 1$ and then replacing $z$ by $x / 2$ throughout to return to $[-2,+2]$. In this case we are working with

$$
\delta_{n}=\max _{0 \leqq k \leqq n+1}\left|x_{k, n}-2 \cos \left(\frac{k \pi}{n+1}\right)\right|
$$

where the $x_{k, n}$ are the Chebyshev alternation points for $f$ on $[-2,+2]$. It is easy to see from this corollary that for $j$ sufficiently large at least $\left(n_{j}+2\right) / 5+2$ of the $n_{j}+2$ alternation points are in the interval $[1,2]$.

For each $n=0,1,2, \ldots$ let $p_{n}$ be the polynomial from $H_{n}$ of best approximation to $f$ on $[-2,2]$. Assume that for $n$ sufficiently large $p_{n}$ is increasing on $[-2,2]$.

We may now use the same argument on $[1,2]$ as is used to arrive at inequality (6) to show that for $j$ sufficiently large

$$
E_{n_{j}}(f) \leqq 5(f(2)-f(1)) /\left(n_{j}+2\right) .
$$

Thus since $f(2)-f(1)=\varepsilon \leqq M / 10$ we have

$$
E_{n_{j}}(f) \leqq 5 \varepsilon /\left(n_{j}+2\right) \leqq M / 2\left(n_{j}+2\right) .
$$

This together with (18) gives for $j$ sufficiently large

$$
M / n_{j} \leqq E_{n_{j}}(f) \leqq M / 2\left(n_{j}+2\right) .
$$

But this implies that $\left(n_{j}+2\right) / n_{j} \leqq \frac{1}{2}$ for $j$ sufficiently large. This is a contradiction since $\lim _{j \rightarrow \infty}\left(n_{j}+2\right) / n_{j}=1$. This proves Theorem 3.1.

4. Conclusions. There is a gap between the main theorems of this paper and the main theorem in [7]. That is, if we assume that $f \in C^{1}[-1,+1]$ and $f^{\prime}(x) \geqq \rho>0$ on $[-1,+1]$ then does it follow that for $n$ sufficiently large the polynomial from $H_{n}$ of best approximation to $f$ is increasing? This author conjectures that the answer is no, but this remains an open question.

The condition on $f^{\prime \prime}$ in Theorem 1.1 can be relaxed, but this theorem will appear elsewhere.

\section{REFERENCES}

1. M. I. Kadec, On the distribution of points of maximum deviation in the approximation of continuous functions by polynomials, Uspehi Mat. Nauk 15 (1960), no. 1 (91), 199-202; English transl., Amer. Math. Soc. Transl. (2) 26 (1963), 231-234. MR 22 \#3920; 27 \#1750.

2. G. G. Lorentz, Approximation of functions, Holt, Rinehart and Winston, New York, 1966. MR 35 \# 4642; erratum, 36, p. 1567.

3. - Monotone approximation, Inequalities III, O. Shisha, Ed., Academic Press, New York, 1969, pp. 201-215.

4. G. G. Lorentz and K. L. Zeller, Monotone approximation by algebraic polynomials, Trans. Amer. Math. Soc. 149 (1970), 1-18. MR 44 \#3060.

5. —, Degree of approximation by monotone polynomials. II, J. Approximation Theory 2 (1969), 265-269. MR 39 \#5991. 
6. J. A. Roulier, Monotone and weighted approximation, Dissertation, Syracuse University, 1968.

7. — Polynomials of best approximation which are monotone, J. Approximation Theory 9 (1973), 212-217.

8. S. B. Stečkin, On the order of the best approximations of continuous functions, Izv. Akad. Nauk SSSR Ser. Mat. 15 (1951), 219-242. (Russian) MR 13, 29.

Department of Mathematics, North Carolina State University, Raleigh, North CaroLINA 27607 